OPEN ACCESS

Edited by:

Wolfgang Kemmler, Friedrich-Alexander-University Erlangen-Nürnberg, Germany

Reviewed by: Michael Bebenek, University of Erlangen Nuremberg, Germany

Marc Teschler, Institute for Rehabilitation Research Norderney, Germany

*Correspondence: Nicolas Wirtz n.wirtz@dshs-koeln.de

Specialty section: This article was submitted to

Exercise Physiology,

a section of the journal

Frontiers in Physiology

Received: 10 April 2019 Accepted: 07 October 2019 Published: 08 November 2019

Citation:

Wirtz N, Dörmann U, Micke F, Filipovic A, Kleinöder $\mathrm{H}$ and Donath $\mathrm{L}$

(2019) Effects of Whole-Body Electromyostimulation on Strength-, Sprint-, and Jump Performance in Moderately Trained Young Adults: A Mini-Meta-Analysis of Five Homogenous RCTs of Our Work Group. Front. Physiol. 10:1336 doi: 10.3389/fphys.2019.01336

\section{Effects of Whole-Body Electromyostimulation on Strength-, Sprint-, and Jump Performance in Moderately Trained Young Adults: A Mini-Meta-Analysis of Five Homogenous RCTs of Our Work Group}

\author{
Nicolas Wirtz*, Ulrike Dörmann, Florian Micke, André Filipovic, Heinz Kleinöder and \\ Lars Donath
}

Department of Intervention Research in Training Science, Institute of Training Science and Sport Informatics, German Sport University Cologne, Cologne, Germany

Background: Whole-body electromyostimulation (WB-EMS) gained increasing interest in sports within recent years. However, few intervention studies have examined the effects of WB-EMS on trained subjects in comparison to conventional strength training.

Objective: The aim of the present mini-meta-analysis of 5 recently conducted and published randomized controlled WB-EMS trails of our work group was to evaluate potentially favorable effects of WB-EMS in comparison to conventional strength training.

Methods: We included parameter of selected leg muscle's strength and power as well as sprint and jump performance. All subjects were moderately trained athletes [ $>2$ training sessions/week, $>2$ years of experience in strength training; experimental group ( $n=$ 58): $21.5 \pm 3.3 \mathrm{y} ; 178 \pm 8 \mathrm{~cm} ; 74.0 \pm 11 \mathrm{~kg}$; control group $(n=54): 21.0 \pm 2.3 \mathrm{y}$; $179.0 \pm 9 \mathrm{~cm} ; 72.6 \pm 10 \mathrm{~kg}$. The following WB-EMS protocols were applied to the experimental group (EG): 2 WB-EMS sessions/week, bipolar current superimposed to dynamic exercises, $85 \mathrm{~Hz}, 350 \mu \mathrm{s}, 70 \%$ of the individual pain threshold amperage. The control groups (CG) underwent the same training protocols without WB-EMS, but with external resistance.

Results: Five extremely homogenous studies (all studies revealed an $l^{2}=0 \%$ ) with 112 subjects in total were analyzed with respect to lower limb strength and power in leg curl, leg extension and leg press machines, sprint-and jump performance. Negligible effects in favor of WB-EMS were found for $F_{\max }$ of leg muscle groups [SMD: 0.11 (90\% Cl: $-0.08,0.33), p=0.73, I^{2}=0 \%$ ] and for CMJ [SMD: 0.01 (90\% Cl: -0.34 , $\left.0.33), p=0.81, l^{2}=0 \%\right]$. Small effects, were found for linear sprint [SMD: $0.22(90 \%$ Cl: $\left.-0.15,0.60), p=0.77, I^{2}=0 \%\right]$ in favor of the EMS-group compared to CON. 


\begin{abstract}
Conclusion: We conclude that WB-EMS is a feasible complementary training stimulus for performance enhancement. However, additional effects on strength and power indices seem to be limited and sprint and jump-performance appear to be benefiting only slightly. Longer training periods and more frequent application times and a slightly larger stimulus could be investigated in larger samples to further elucidate beneficial effects of WB-EMS on performance parameters in athletes.
\end{abstract}

Keywords: WB-EMS, electrical stimulation, strength training, MVC, peak power output

\section{INTRODUCTION}

Electromyostimulation (EMS) is a common and established method to enhance muscular strength and performance. Systematic reviews have well documented beneficial influence of locally applied EMS on strength (Delitto et al., 1989; Bax et al., 2005; Requena Sánchez et al., 2005; Paillard, 2008; Filipovic et al., 2012) and the neuromuscular parameters (Vanderthommen and Duchateau, 2007). Further studies revealed positive effects on jump and sprint capacity (Wolf et al., 1986; Brocherie et al., 2005; Herrero et al., 2006, 2010a,b; Babault et al., 2007; Maffiuletti et al., 2009; Billot et al., 2010; Voelzke et al., 2012; Filipovic et al., 2016; Wirtz et al., 2016). The reasons for the improvements using EMS are a higher number of motor units recruited during exercise with EMS compared to voluntary dynamic contractions only (Kots and Chwilon, 1971). Additionally, the activation of fasttwitch fibers at relatively low force levels plays also a relevant role (Gregory and Bickel, 2005). Most studies used the maximum pain threshold (maximum tolerated amperage) to regulate the impulse intensity (amperage) (Brocherie et al., 2005; Maffiuletti et al., 2009). However, a high level of muscle tension due to EMS limits the range of dynamic movements. Therefore, in dynamic exercise modes with superimposed EMS, the impulse intensity need to be adjusted to ensure sufficient movement. 70\% of maximum pain threshold is considered practicable and might be more promising, as the subjective feeling of increased remains comfortable (Wirtz et al., 2016; Micke et al., 2018). Dynamic movements with additional EMS can also increase activation levels at different muscle length and during different contraction modes, e.g., during eccentric work phases (Westing et al., 1990). Authors hypothesized that type II muscle fibers remain active during EMS in contrast to the normal continuing de-recruitment of motor units during the eccentric phase. Therefore, the intensification of exercise by superimposed EMS can potentially induce an increase in recruitment of high-threshold motor units.

Technical innovations made EMS progress from a local stimulation to a whole-body training method where several muscle groups can be trained simultaneously through an electrode belt- and vest system (e.g., miha bodytec, Augsburg, Germany). Improved handling and simplified use led to increased recognition of whole-body-EMS (WB-EMS) training for coaches and athletes. Today WB-EMS is used in leisure sports and showed effects in both individual sports (AmaroGahete et al., 2018) and field sports on a high-performance level (Filipovic et al., 2016). WB-EMS enables the activation of several muscle groups simultaneously, e.g., muscle chains or agonist/antagonist during multi joint movements. This allows to train strength exercise like squats or sport specific skill exercises like jumps with superimposed WB-EMS that may support a strength transfer to more complex movements.

Strength and performance adaptations are evident for trained subjects by the use of local EMS (Filipovic et al., 2012) and for untrained subjects using WB-EMS (Kemmler et al., 2018). There is however a lack of studies including performance parameters of trained subjects. Therefore, the aim of this study is to provide evidence for the effect of training with superimposed WB-EMS on lower leg strength and power as well as sprint and jump performance in trained subjects. In this regard we conducted a mini-meta-analysis focusing on individual data of 5 recent in-house WB-EMS studies (Dörmann, 2011; Wirtz et al., 2016; Micke et al., 2018; Dörmann et al., 2019; Filipovic et al., 2019). All studies were designed to characterize the impact of superimposed WB-EMS during different exercise conditions. Exercises were designed to improve strength and power of leg muscle chain and to improve jump and sprint performance. All studies included the outcome parameter strength and power of certain leg muscle groups. Furthermore, a high standardization of EMS-adjustments characterizes all studies. Our primary hypothesis was that superimposed WB-EMS favors strength and power of lower limb muscles significantly. Our secondary hypothesis was that WB-EMS favors jumping and sprinting performance significantly.

\section{MATERIALS AND METHODS}

\section{Participants}

One hundred and twelve male (68\%) and female (32\%) subjects were included into this mini-meta-analysis $(n=112$; participants characteristics are presented in Table 1). All subjects were moderately trained doing $>2$ training sessions/week on a regional to national level in sports that require sprint and/or jump performances (e.g., soccer, handball, basketball, track, and field, tennis). They were examined medically and signed a written consent about the possible risks and benefits of the study. Exclusion criteria were (a) planned absences during the whole study period, (b) any training experience in WB-EMS, (c) current training programs focusing on sprinting and jumping as well as, (d) inadequate technique in the strength exercises. In order to minimize influences of unspecific training loads, all participants were asked to refrain from any changes of their habitual physical activity behavior. Furthermore, all participants were instructed to maintain their normal dietary intake before and during the study. 
TABLE 1 | Anthropometric data (mean \pm SD).

\begin{tabular}{|c|c|c|c|c|c|c|}
\hline & $\begin{array}{c}N \\
\text { (male/female) }\end{array}$ & $\begin{array}{c}\text { Age } \\
\text { [years] }\end{array}$ & $\begin{array}{l}\text { Height } \\
\text { [cm] }\end{array}$ & $\begin{array}{l}\text { Weight } \\
\text { [kg] }\end{array}$ & $\begin{array}{c}\text { BMI } \\
{\left[\mathrm{kg} / \mathrm{m}^{2}\right]}\end{array}$ & $\begin{array}{c}\text { Strength training experience } \\
\text { [years] }\end{array}$ \\
\hline EG & $\begin{array}{c}58 \\
(39 / 19)^{a}\end{array}$ & $21.5 \pm 3.3$ & $178.2 \pm 7.5$ & $74.0 \pm 11.2$ & $23.2 \pm 2.5$ & $5.4 \pm 3.7$ \\
\hline CG & $\begin{array}{c}54 \\
(37 / 17)^{b}\end{array}$ & $21.0 \pm 2.3$ & $179.0 \pm 8.5$ & $72.6 \pm 9.8$ & $22.6 \pm 2.1$ & $4.4 \pm 2.9$ \\
\hline
\end{tabular}

a Dörmann et al. (2011) (5/2); Dörmann et al. (2019) (0/10); Micke et al. (2018) (14/7); Filipovic et al. (2019) (10/0); Wirtz et al. (2016) (10/0).

${ }^{b}$ Dörmann et al. (2011) (5/2); Dörmann et al. (2019) (0/11); Micke et al. (2018) (12/4); Filipovic et al. (2019) (10/0); Wirtz et al. (2016) (10/0).

The study protocols were approved by the "Ethics Committee of the German Sport University Cologne" and complied with the Declaration of Helsinki "Ethical Principles for Medical Research Involving Human Subjects.”

\section{Study Design}

The aim of the current mini-meta-analytical review was to compare the pooled favorable effects of submaximal, superimposed dynamic WB-EMS (EG) with the effects of dynamic athletic training without WB-EMS (CG) on (1) strength and power performance as well as on (2) sprinting and jumping performance. To adequately address our hypothesis we conducted individual data analysis derived from 5 randomized controlled trials (RCT) with parallel group designs (WB-EMS vs. active control) carried out between 2010 and 2017 by the Institute of Training Science and Sport Informatics, German Sport University Cologne, Germany. For the present metaanalysis, we initially selected in-house studies that (1) included trained subjects aged 18-30 years training on regional to national level and had at least 2 years of strength training experience but NO previous WB-EMS experience; (2) applied a randomized controlled trial (RCT) approach with parallel group designs (WBEMS vs. active control); randomization by minimization method (strata: age, gender, strength training experience); (3) applied a WB-EMS protocol for $4-8$ weeks with 2 training sessions per week; (4) conducted the same test settings for sprint, jump, power and strength diagnostics. Eligibility and study quality [Physiotherapy Evidence Database (PEDro) scale] were assessed (Table 2).

\section{Training Procedure}

For detailed training procedures of the single trials, the reader is kindly referred to the corresponding studies. Studies conducted squats (Wirtz et al., 2016), squats and lunges (Dörmann et al., 2011), squat jumps (Filipovic et al., 2019) or different strength and conditioning exercises such as squats, lunges, nordic curl, skippings, heelings, lateral, horizontal, and vertical jumps (Micke et al., 2018; Dörmann et al., 2019) (Table 3). These exercises followed the recommendations to increase sprinting and jumping performance (Young, 2006; Stojanovic et al., 2017).

All studies implemented an active control group that completed the same exercise protocol without superimposed WB-EMS. All studies had a standardization procedure using the same parameters for both tested groups (EG and CG) like exercises, number of repetitions, number of sets, range of motion, movement velocity, and rate of perceived exertion. In general, all participants completed 8-16 training sessions during a 4-8 week period conducting 2 training sessions per week with a total intervention time under tension between 32 and $113 \mathrm{~min}$.

All WB-EMS interventions complied with the guidelines for a safe and effective WB-EMS training (Kemmler et al., 2016). The miha bodytec system (Augsburg, Germany) was employed as EMS device (cf. Kemmler et al., 2012). The application unit was connected via electrical cords to a stimulation vest and belts. Thereby, bilaterally paired surface electrodes were integrated. 8 muscle areas could be stimulated synchronously with freely selectable impulse intensities (0-120 mA) for each pair of electrodes. In our studies, 3 paired electrodes were applied around the muscle belly of the lower legs $(27 \mathrm{~cm}$ length $\times 4 \mathrm{~cm}$ width), the thighs $(44 \times 4 \mathrm{~cm})$ and at the buttocks $(13 \times 10 \mathrm{~cm})$. Additionally, the upper body was stimulated with 2 bilaterally paired electrodes integrated in the stimulation vest at the lower back $(14 \times 11 \mathrm{~cm})$ and abdominals $(23 \times 10 \mathrm{~cm})$.

The WB-EMS adjustments as well as the progression for the conventional strength and conditioning programs were equal for all interventions. The intensity of each exercise set was controlled by Borg Rating of Perceived Exertion (RPE) (Tiggemann et al., 2010). If a set was no longer exhaustive (RPE $<16$ "hard") the intensity was raised and by increasing additional loads or the use of stiffer rubber bands and by higher amperage for EG. The WB-EMS impulse frequency was set at $85 \mathrm{~Hz}$, the impulse width at 350-400 $\mu \mathrm{s}$, the impulse type as bipolar and rectangle. The intensity of WB-EMS was adjusted to $70 \%$ of the individual pain threshold (iPT $=$ maximum tolerated amperage, $0-120 \mathrm{~mA}$ ). The iPT was verified separately for each pair of electrodes before each session. The participants stood with an inner knee angle of $170^{\circ}$ while tensing their muscles voluntary. The verification of iPT began increasing current to iPT at the buttock, followed by the thigh, the lower leg, the abdominal, and the lower back. Then, the intensity was subsequently downregulated using the main controller at the WB-EMS device to an intensity of $70 \%$ to enable dynamic movements.

\section{Testing Procedure Strength and Power Testing}

Strength and power diagnostics took place on the Leg Curl (LC), the Leg Extension (LE), and the Leg Press (LP) machine (EditionLine, gym80; Gelsenkirchen, Germany). Those were equipped with the digital measurement equipment Digimax (mechaTronic; Hamm, Germany). The software IsoTest and DynamicTest 2.0 


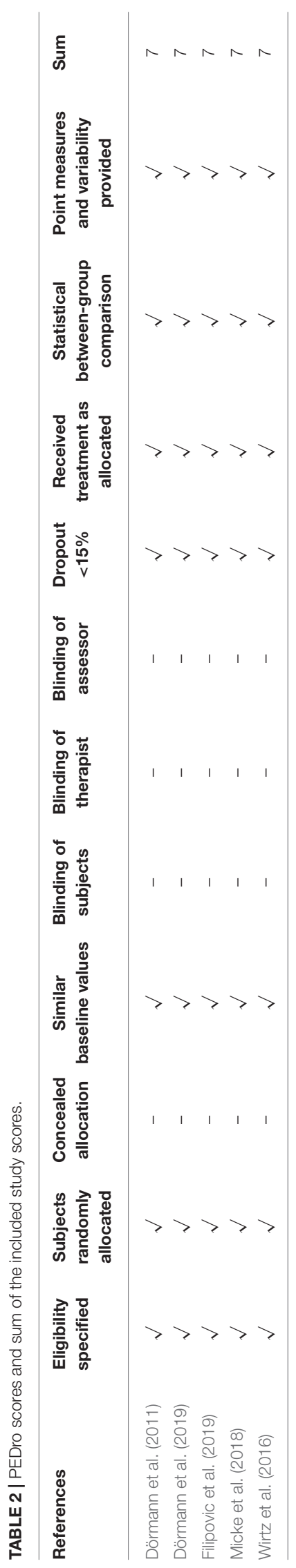

enabled the measurement of the peak force $\mathrm{F}_{\max }$ and the peak power $\mathrm{P}_{\max }(5 \mathrm{kN}$ strength sensor typ KM1506, distance sensor typ S501D, megaTron; Munich, Germany). The sensors were installed in line with the steel belt of the machines that lifts the selected weight plates.

Diagnostic procedures consisted of 3 isometric trials as well as 3 isoinertial trials on LE, LC, and LP. Isometric attempts were conducted at an inner knee angle of $120^{\circ}$ on LE and LP as well as of $150^{\circ}$ on LC. The instruction was to press as forcefully and as fast as possible against the fixed lever arm. This enabled the determination of knee joint angle-dependent forcetime curve during explosive maximum voluntary contraction. Concerning isoinertial tests, the participants were requested to move the lever arm as forcefully and as fast as possible over the complete concentric range of motion (ROM). This enabled the examination of knee joint angle-dependent power-load curve during explosive maximum voluntary leg extension on LP, knee extension on LE or knee flexion on LC. The concentric ROM corresponded to $90-180^{\circ}$ inner knee angles on LP and LE as well as to $170-80^{\circ}$ inner knee angles on LC. Additional load (AL) was calculated individually as a percentage of an isometric attempt at starting position of isoinertial tests. These were $90^{\circ}$ on LP and LE as well as $170^{\circ}$ on LC. Three attempts were conducted with $40 \% \mathrm{AL}$ on LE and LC as well as 3 attempts with $60 \%$ $\mathrm{AL}$ on LP. The rest was defined as 60 s between every single trial and $3 \mathrm{~min}$ between the different test types. The parameters $\mathrm{F}_{\max }[\mathrm{N}]$ and $\mathrm{P}_{\max }[\mathrm{W}]$ were calculated for statistical analysis and data presentation as best performance data. Reliability was determined by the coefficient of variation $(\mathrm{CV})$ and the intraclass correlation coefficient (ICC) for parameters force $(\mathrm{F})(\mathrm{CV}<8 \%$; ICC $0.95-0.97$ ), as well as for power (P) and power factors (F.V) (CV < 9\%; ICC 0.84-0.97) for all used machines (Dörmann, 2011).

\section{Sprint Testing}

Sprint testing involved a $20 \mathrm{~m}$ sprint. The test was performed with a self-initiated standing start with no hopping or backward movement prior the start. Double infrared photoelectric barriers (DLS/F03, Sportronic; Leutenbach-Nellmersbach, Germany) were used to measure the time. The best sprinting time out of 2 attempts was used for subsequent analysis. The participants had 2 min rest between the trials. Sprint running performance tests (linear and change of direction) were shown as highly relative reliable (CV 1-6\%; ICC 0.80-0.96) (Green et al., 2011).

\section{Jump Testing}

Following one familiarization jump trial, the participants performed 3 counter movement jumps (CMJ). The participants were instructed to start jumping from an upright standing position, squatting down to a knee angle of approximately $90^{\circ}$ in order to jump as high as possible. Hands had to remain in the akimbo position for the entire movement of each jump to minimize the influence of arm swing. The highest jump was used for subsequent analysis. The Optojump system (Microgate; Bolzano, Italy) was used to verify jump height by the flight time method. It is based on measurements of optical light emitting diodes. Optojump based jump height was 
TABLE 3 | Overview of the included studies

\begin{tabular}{|c|c|c|c|c|c|c|c|}
\hline References & Study design & $\begin{array}{l}\text { Sample: population; } \\
\text { sample size }(n) ; \text { age, } \\
\text { y (mean } \pm \text { SD) }\end{array}$ & Groups & Intervention & Training characteristics & Outcome measures & $\begin{array}{l}\text { Study quality } \\
\text { (PEDro score) }\end{array}$ \\
\hline $\begin{array}{l}\text { Dörmann } \\
\text { et al. (2011) }\end{array}$ & $\begin{array}{l}\text { Randomized controlled } \\
\text { trial, two arms }\end{array}$ & $\begin{array}{l}\text { Healthy sport students, } \\
n=14 \\
21.3 \pm 1.7 \text { y }\end{array}$ & $\begin{array}{l}\mathrm{EG}(n=7) \\
\mathrm{CG}(n=7)\end{array}$ & $\begin{array}{l}\text { Supervised training: } \\
\text { (a) WB-EMS superimposed to squat } \\
\text { exercises } \\
\text { (b) Squat exercise with additional loads } \\
\text { (10RM) }\end{array}$ & $\begin{array}{l}4 \text { weeks, } 2 \text { sessions/week; } \\
3 \text { exercises/session, } 3 \text { sets, total } \\
\text { net exercise time: } 72 \text { min }\end{array}$ & $\begin{array}{l}F_{\max } \text { and } P_{\max } \text { at Leg Curl and } \\
\text { Leg Press Machine; Linear Sprint } \\
\text { time; counter movement jump } \\
\text { height }\end{array}$ & 7 \\
\hline $\begin{array}{l}\text { Dörmann } \\
\text { et al. (2019) }\end{array}$ & $\begin{array}{l}\text { Randomized controlled } \\
\text { trial, two arms }\end{array}$ & $\begin{array}{l}\text { Healthy sport students, } \\
n=21 \\
19.7 \pm 1.7 \text { y }\end{array}$ & $\begin{array}{l}\mathrm{EG}(n=10) \\
\mathrm{CG}(n=11)\end{array}$ & $\begin{array}{l}\text { Supervised training: } \\
\text { (a) WB-EMS superimposed to squat } \\
\text { exercises, nordic curl, sprint and jump } \\
\text { training } \\
\text { (b) Squat exercise (10 RM), sprint and } \\
\text { jump training }\end{array}$ & $\begin{array}{l}4 \text { weeks, } 2 \text { sessions/week; } \\
4-5 \text { exercises/session, } 3 \text { sets, } \\
\text { total net exercise time: } 91 \text { min }\end{array}$ & $\begin{array}{l}F_{\max } \text { and } P_{\max } \text { at Leg Curl, Leg } \\
\text { Extension and Leg Press } \\
\text { Machine; Linear Sprint time; } \\
\text { counter movement jump height }\end{array}$ & 7 \\
\hline $\begin{array}{l}\text { Filipovic } \\
\text { et al. (2019) }\end{array}$ & $\begin{array}{l}\text { Randomized controlled } \\
\text { trial, two arms }\end{array}$ & $\begin{array}{l}\text { Healthy sport students, } \\
n=20 \\
24.4 \pm 4.0 \text { y }\end{array}$ & $\begin{array}{l}\mathrm{EG}(n=10) \\
\mathrm{CG}(n=10)\end{array}$ & $\begin{array}{l}\text { Supervised training: } \\
\text { (a) WB-EMS superimposed to jump } \\
\text { training } \\
\text { (b) Jump training }\end{array}$ & $\begin{array}{l}8 \text { weeks, } 2 \text { sessions/week; } \\
1 \text { exercise/session, } 3 \text { sets, total } \\
\text { net exercise time: } 32 \text { min }\end{array}$ & $\begin{array}{l}F_{\max } \text { and } P_{\max } \text { at Leg Curl, Leg } \\
\text { Extension, and Leg Press } \\
\text { Machine; counter movement } \\
\text { jump height }\end{array}$ & 7 \\
\hline $\begin{array}{l}\text { Micke et al. } \\
\text { (2018) }\end{array}$ & $\begin{array}{l}\text { Randomized controlled } \\
\text { trial, two arms }\end{array}$ & $\begin{array}{l}\text { Healthy sport students, } \\
n=37 \\
20.8 \pm 2.1 \text { y }\end{array}$ & $\begin{array}{l}\mathrm{EG}(n=21) \\
\mathrm{CG}(n=16)\end{array}$ & $\begin{array}{l}\text { Supervised training: } \\
\text { (a) WB-EMS superimposed to squat } \\
\text { exercises, Nordic curl, sprint and jump } \\
\text { training } \\
\text { (b) Squat exercises, Nordic curls, sprint } \\
\text { and jump training }\end{array}$ & $\begin{array}{l}8 \text { weeks, } 2 \text { sessions/week; } \\
4-5 \text { exercises/session, } 3 \text { sets, } \\
\text { total net exercise time: } 113 \text { min }\end{array}$ & $\begin{array}{l}F_{\max } \text { and } P_{\max } \text { at Leg Curl, Leg } \\
\text { Extension, and Leg Press } \\
\text { Machine; Linear Sprint time; } \\
\text { counter movement jump height }\end{array}$ & 7 \\
\hline $\begin{array}{l}\text { Wirtz et al. } \\
\text { (2016) }\end{array}$ & $\begin{array}{l}\text { Randomized controlled } \\
\text { trial, two arms }\end{array}$ & $\begin{array}{l}\text { Healthy sport students, } \\
n=20 \\
22.1 \pm 1.9 y\end{array}$ & $\begin{array}{l}\mathrm{EG}(n=10) \\
\mathrm{CG}(n=10)\end{array}$ & $\begin{array}{l}\text { Supervised training: } \\
\text { (a) WB-EMS superimposed to squat } \\
\text { exercises with additional loads (10 RM) } \\
\text { (b) Squat exercise with additional loads } \\
\text { (10 RM) }\end{array}$ & $\begin{array}{l}6 \text { weeks, } 2 \text { sessions/week; } 1 \\
\text { exercise/session, } 4 \text { sets, total } \\
\text { net exercise time: } 48 \text { min }\end{array}$ & $\begin{array}{l}F_{\max } \text { and } P_{\max } \text { at Leg Curl, Leg } \\
\text { Extension, and Leg Press } \\
\text { Machine; Linear Sprint time; } \\
\text { counter movement jump height }\end{array}$ & 7 \\
\hline
\end{tabular}




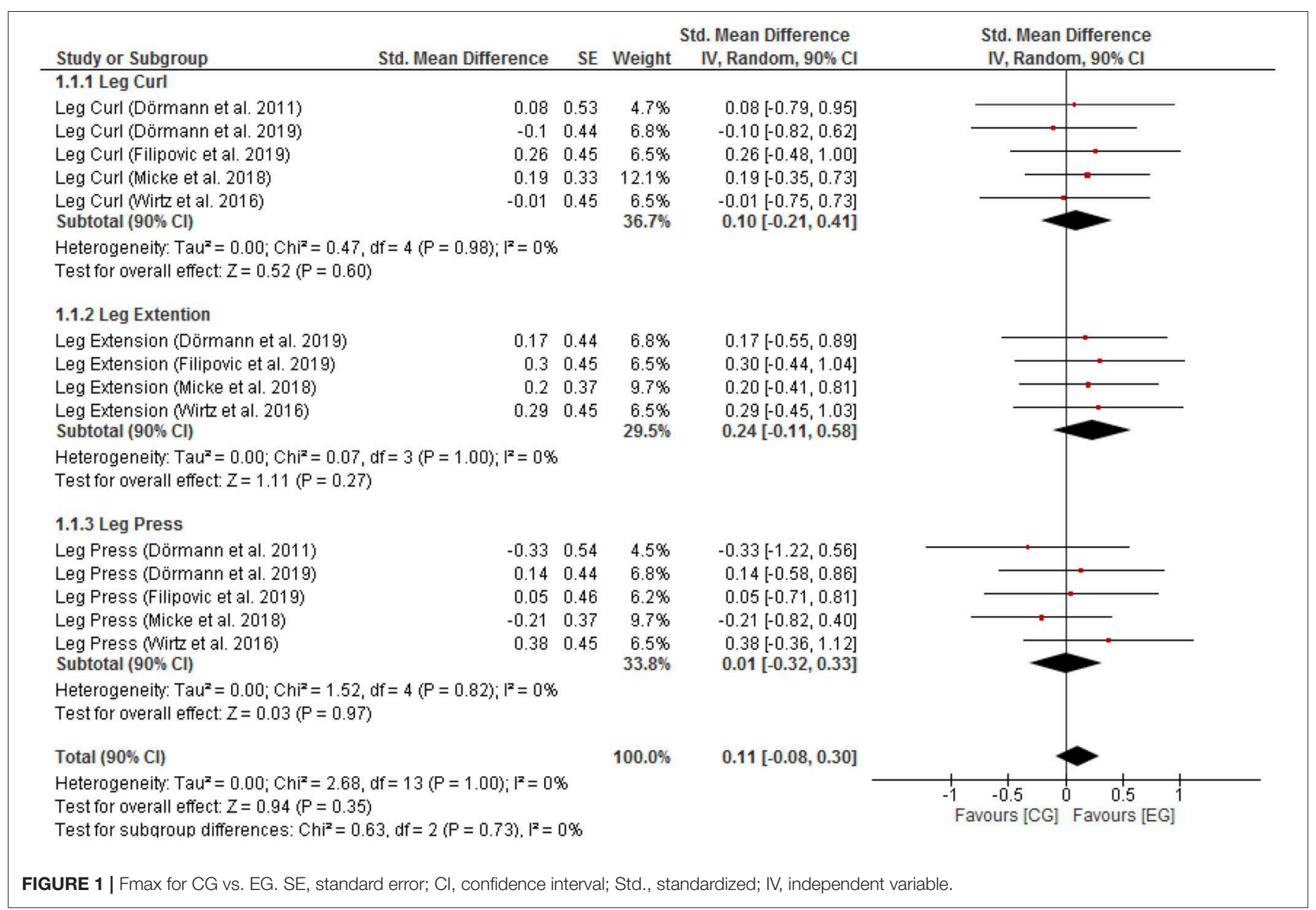

shown as highly relative reliable $(\mathrm{CV}<3 \%$ and $\mathrm{ICC}>0.9)$ (Glatthorn et al., 2011).

\section{Statistical Analysis}

Standardized mean differences (SMD, with 90\% confidence intervals) were computed for each study using the adjusted Hedges' g (Equation 1). This adjustment takes small sample biases into account. The Cochrane Review Manager Software (RevMan 5.3.5, Cochrane Collaboration, Oxford, UK) was used to compute the inverse-variance method according to Deeks and Higgins (2010). Analyses were conducted with a random effects model (Borenstein et al., 2010). Forest plots were generated for each outcome category (performance, physical performance surrogates and psychological variables). The magnitude of effect sizes was classified according to the following scale: $0-0.19=$ negligible effect, $0.20-0.49=$ small effect, $0.50-0.79=$ moderate effect and $0.80=$ large effect (Cohen, 1988).

Equation 1: Equation to calculate standardized mean differences (SMD) adjusting for small sample sizes.

$$
S M D_{i}=\frac{m_{1 i}-m_{2 i}}{s_{i}}\left(1-\frac{3}{4 N_{i}-9}\right)
$$

\section{RESULTS}

\section{Strength and Power}

Negligible effects with low heterogeneity were found for $F_{\max }$ leg muscle groups [SMD: 0.11 (90\% CI: $-0.08,0.33), p=0.73$, $I^{2}=0 \%$; Figure 1)]. Negligible effects with low heterogeneity were also found for $\mathrm{P}_{\max }$ leg muscle groups [SMD: 0.12 (90\% CI: $-0.07,0.30), p=0.90, I^{2}=0 \%$; Figure 2].

\section{Linear Sprint}

Small effects with low heterogeneity were found for linear sprint [SMD: 0.22 (90\% CI: $-0.15,0.60), p=0.77, I^{2}=0 \%$; Figure 3] in favor of EG to CG.

\section{Counter Movement Jump}

Neglibible effects with low heterogeneity were found for CMJ height [SMD: 0.01 (90\% CI: $-0.34,0.33), p=0.81, I^{2}=0 \%$; Figure 4]. Data of all analyzed parameter are summarized in supplementary material (Data Sheet 1-4).

\section{DISCUSSION}

This meta-analysis investigated the pooled effect sizes of superimposed WB-EMS in comparison to conventional strength and conditioning training on (1) strength and power of 


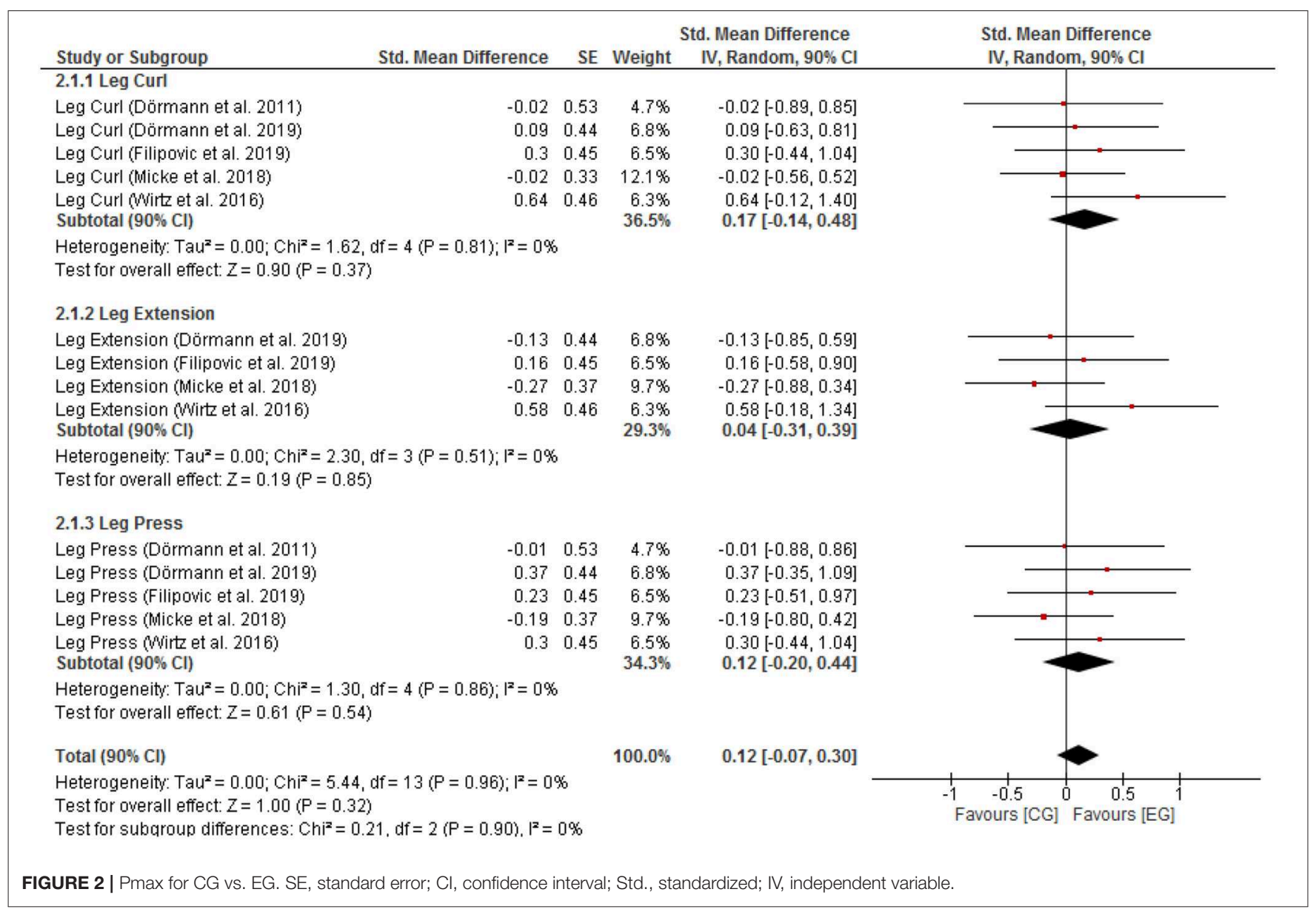

\begin{tabular}{|c|c|c|c|c|c|c|c|}
\hline \multirow{2}{*}{$\begin{array}{l}\text { Study or Subgroup } \\
\text { Linear Sprint (Dörmann et al. 2011) }\end{array}$} & Std. Mean Difference & SE & \multirow{2}{*}{ Weight } & \multirow{2}{*}{$\begin{array}{r}\text { Std. Mean Difference } \\
\text { IV, Random, } 90 \% \mathrm{Cl}\end{array}$} & \multicolumn{2}{|c|}{$\begin{array}{l}\text { Std. Mean Difference } \\
\text { IV, Random, } 90 \% \mathrm{Cl}\end{array}$} & \\
\hline & -0.27 & 0.54 & & & $=-$ & & \\
\hline Linear Sprint (Micke et al. 2018) & 0.25 & 0.42 & $29.5 \%$ & $0.25[-0.44,0.94]$ & & $\longrightarrow$ & \\
\hline Linear Sprint (Wirtz et al. 2016) & 0.46 & 0.45 & $25.7 \%$ & $0.46[-0.28,1.20]$ & & & \\
\hline LinearSprint (Dörmann et al. 2019) & 0.29 & 0.44 & $26.9 \%$ & $0.29[-0.43,1.01]$ & & 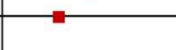 & - \\
\hline Total $(90 \% \mathrm{Cl})$ & & & $100.0 \%$ & $0.22[-0.15,0.60]$ & & & \\
\hline \multicolumn{3}{|c|}{$\begin{array}{l}\text { Heterogeneity: } \mathrm{Tau}^{2}=0.00 ; \mathrm{Ch}^{2}=1.14, \mathrm{df}=3(\mathrm{P}=0.77) ; \mathrm{I}^{2}=0 \% \\
\text { Test for owerall effect: } Z=0.97(\mathrm{P}=0.33)\end{array}$} & & & $\begin{array}{ccc}+ & -1 \\
-1 & -0.5 & 0 \\
& \text { Favours [CG] }\end{array}$ & $\begin{array}{cc}0 & 0.5 \\
\text { Favours [EG] }\end{array}$ & 1 \\
\hline
\end{tabular}

\begin{tabular}{|c|c|c|c|c|c|}
\hline Study or Subgroup & Std. Mean Difference & SE & Weight & $\begin{array}{l}\text { Std. Mean Difference } \\
\text { IV, Random, } 90 \% \mathrm{Cl}\end{array}$ & $\begin{array}{l}\text { Std. Mean Difference } \\
\text { IV, Random, } 90 \% \mathrm{Cl}\end{array}$ \\
\hline CMJ (Dörmann et al. 2019) & -0.18 & 0.44 & $21.8 \%$ & $-0.18[-0.90,0.54]$ & \begin{tabular}{l|l} 
& \\
\end{tabular} \\
\hline CMJ (Filipovic et al. 2019) & 0.34 & 0.45 & $20.8 \%$ & $0.34[-0.40,1.08]$ & \\
\hline CMJ (Wicke et al. 2018) & -0.15 & 0.34 & $36.5 \%$ & $-0.15[-0.71,0.41]$ & $\longrightarrow$ \\
\hline CMJ (Wirt et al. 2016) & 0.08 & 0.45 & $20.8 \%$ & $0.08[-0.66,0.82]$ & \\
\hline Total $(90 \% \mathrm{Cl})$ & & & $100.0 \%$ & $-0.01[-0.34,0.33]$ & \\
\hline \multicolumn{5}{|c|}{$\begin{array}{l}\text { Heterogeneity: } \text { Tau }^{2}=0.00 ; \mathrm{Chi}^{2}=0.96, \mathrm{df}=3(\mathrm{P}=0.81) ; \mathrm{I}^{2}=0 \% \\
\text { Test for overall effect: } Z=0.03(\mathrm{P}=0.97)\end{array}$} & $\begin{array}{ccccc}1 & 1 & 1 & 1 & 1 \\
-1 & -0.5 & 0 & 0.5 & 1 \\
\text { Favours [CG] } & \text { Favours [EG] }\end{array}$ \\
\hline
\end{tabular}


lower limb muscles as well as on (2) jumping and sprinting performance. Our analyses rely on findings of five homogenous studies of overall high study quality of our work group. It was hypothesized that additional superimposed WB-EMS may lead to favorable strength and power improvements of lower limb muscles as well as increases in jumping and sprinting performance compared to training without WB-EMS.

The main findings indicate that superimposed WB-EMS did not lead to superior effects of strength and power of lower limb muscle groups and jumping performance. Interestingly, sprint performance benefited with small but meaningful effects when applying WB-EMS. Generally, PEDro score analyses of the included studies revealed a "low risk of bias." Blinding of participants and personnel is difficult in exercise studies in general and WB-EMS investigation in particular. Promoting evidence-based WBEMS training programs requires studies that consider intention to treat analysis, blinding assessors, and reporting the risk of co-interventions. Future studies should account for these quality criteria.

Our pooled data analyses do not corroborate superior effects of WB-EMS on certain muscle groups. A small effect was merely observed for $\mathrm{F}_{\max }$ for leg extension, but not for leg curl and leg press or LP. These results are surprising as leg extension exercise, such as squats were included in each intervention in both groups (EG and CG). The application of superimposed WB-EMS might beneficially affect maximal strength of the quadriceps muscles when additional motor unit recruitment with (a) a continuous and exhausting contractile activity in the same pool of motor units during the entire exercise period, (b) a supramaximal temporal recruitment imposed by the high stimulation frequency chosen, and (c) a synchronous recruitment of neighboring muscle fibers might account for these strength gains (Requena Sánchez et al., 2005) is guaranteed. Nevertheless, the overall effects are close to the zero-line of the forest plot and it would be speculative to assume that a higher sample sizes or longer application time lead to differential results. However, one study showed muscle group-specific adaptations of hamstring power after squats with superimposed WB-EMS (Wirtz et al., 2016). Despite notable co-activation (Zink et al., 2001), it has been reported that the activation of hamstring muscles is not affected by additional loading during squat exercise (Nuzzo and McBride, 2013). EMS can lead to higher hamstring muscle activation during lengthening and shortening, which would then results in higher hamstring maximum force in the WB-EMS training group. One potential explanation for these inconsistent results could be that the different underlying exercises and populations of the included studies, such as squats (Wirtz et al., 2016), jumps (Filipovic et al., 2019) or different strength and conditioning hamstring exercises (Micke et al., 2018) increase variability of the occurrence and magnitude of potential effects. Available evidence on the adaptability of the hamstrings employing WB-EMS independent from the type of exercise is needed and would provide specific insights into WBEMS use to strengthen the hamstrings and to possibly prevent injuries. Particularly hamstring strains are reported to be the most prevalent muscle injury in various team and sprint related sports (Goode et al., 2015).
The simultaneous activation of (1) multiple muscle groups and (2) agonistically and antagonistically working muscles through WB-EMS combined with strength and training exercises was repeatedly reported to have the potential to improve sport-specific skills such as sprinting and jumping. Although WB-EMS triggers a seemingly counterproductive firing of the agonist and antagonist, a voluntary contraction reduces relative co-activation of antagonistic muscles, in order to continue the required dynamic exercise. Indeed, available data do not suggest such effects during superimposed WB-EMS for jumping performance. Thus, it might be beneficial to focus on specific jump exercise with superimposed EMS (Filipovic et al., 2019). In line with this reasoning, one study with professional soccer players revealed that jumps with superimposed WB-EMS in addition to soccer training sessions can be effective for jump improvements (Filipovic et al., 2016). Overall, it appears to be plausible to assume that exercise specificity and the training status of the participants affect the effects of superimposed WB-EMS. Potential improvements by the use of maximal and locally applied EMS also rely on training specificity, such as combined plyometric training (Herrero et al., 2006) and sport specificity on a higher level in sports with numerous jumps like volleyball (Malatesta et al., 2003; Voelzke et al., 2012) or basketball (Maffiuletti et al., 2000). For sprinting performance, however, minor effects could be found. The results of linear sprint are in accordance with studies that applied isometric local EMS with additional separately performed strength and sprint training sessions: One study reported improvements of $10 \mathrm{~m}$ skating time for $2^{\text {nd }}$ league ice hockey players, who trained on ice parallel to training intervention, what could elicited utilization effects (Brocherie et al., 2005). It is however reasonable that effects of WB-EMS on sprinting performance as well as jumping performance could depend on athletes' training status.

However, some limitations of the present study need to be addressed that should be considered for further research on WBEMS. One is seen in the small sample sizes of each included studies. However, outcomes and assessment over all studies are very homogenous. Although we intended to increase the cumulative power by pooling the data, a compiled and robust effect cannot be found. Although minor improvement in top level athletes can be considered relevant, negligible additional benefits from WB-EMS in comparison to conventional training can be summarized. Ultimately, WB-EMS provides a variety of different training stimuli but a notable transfer of the results to top level athletes is speculative. Furthermore, the lack of data after a detraining phase hamper an identification of delayed effects that could potentially occur. The adaptations of WB-EMS over time need to be further investigated and concepts for periodization in high performance sports also including WB-EMS need to be developed. A further limitation is seen in the inclusion of both genders. Although Maffiuletti et al. (2008) demonstrated that supra-motor thresholds were significantly lower in women than in men, contrary to the expected constitutional differences like subcutaneous fat thickness, women showed no significant differences at motor threshold. However, the subjective tolerance to current intensity remains a key limiting factor of WB-EMS, regardless of sexes (Gregory and Bickel, 2005). Nevertheless, we 
did not focus on sex differences in trainability. Further limitations are seen in the different designs for lower limb exercises in the studies and the different intervention duration (4-8 weeks) that result in 8-16 total training sessions. However, 4-8 weeks can be regarded as a common and reasonable meso-cycle within periodization considerations.

Only few drop-outs occurred independent of the WB-EMS intervention and an attendance rate of $100 \%$ for all of the 112 included participants was observed and no adverse event was reported. Current intensities around $70 \%$ of maximum pain threshold that enables movement complied with the safety recommendations published by Kemmler et al. (2016). This is particularly important with regard to cases of rhabdomyolysis after WB-EMS training at maximum intensity with professional soccer players (Kastner et al., 2015).

Finally, we can conclude that WB-EMS is a feasible complementary training stimulus for performance enhancement. Additional effects of WB-EMS on relevant strength, power and performance indices seem limited. Longer training periods and more frequent application times and a slightly larger stimulus could be investigated in larger studies in order to further elucidate beneficial effects of WB-EMS on crucial performance parameters in athletes.

\section{DATA AVAILABILITY STATEMENT}

The raw data supporting the conclusions of this manuscript will be made available by the authors, without undue reservation, to any qualified researcher.

\section{AUTHOR CONTRIBUTIONS}

$\mathrm{UD}, \mathrm{NW}, \mathrm{FM}, \mathrm{AF}$, and $\mathrm{HK}$ conceived and designed research. $\mathrm{UD}, \mathrm{NW}, \mathrm{FM}$, and AF conducted experiments. UD, LD, and NW analyzed data. NW, LD, and UD wrote the manuscript. HK, UD,
NW, FM, AF, and LD revised the manuscript. All authors read and approved the manuscript.

\section{FUNDING}

Three of the studies under consideration were funded by the German Federal Institute of Sport Science on behalf of the Federal Ministry of the Interior of Germany as the major funding bodies (Wirtz et al., 2016: BISp AZ 070509/13; Micke et al., 2018: ZMV I 1-070506/14-16; Filipovic et al., 2019: ZMV I 4070101/16/17). All studies were funded by the Department of Intervention Research in Exercise Training, Institute of Exercise Training and Sport Informatics, German Sport University Cologne, Cologne, Germany.

\section{ACKNOWLEDGMENTS}

The authors would like to acknowledge the time and effort of all participants involved in this investigation.

\section{SUPPLEMENTARY MATERIAL}

The Supplementary Material for this article can be found online at: https://www.frontiersin.org/articles/10.3389/fphys. 2019.01336/full\#supplementary-material

Data Sheet 1 | Fmax pre-post in Leg Curl (LC), Leg Extension (LE), and Leg Press (LP) for CG and EG (mean, standard deviation, difference pre-post in \%, effect sizes pre-post and standard error).

Data Sheet 2 | Pmax pre-post in Leg Curl (LC), Leg Extension (LE), and Leg Press (LP) for CG and EG (mean, standard deviation, difference pre-post in \%, effect sizes pre-post and standard error).

Data Sheet 3 | Sprint time 20 m pre-post for CG and EG (mean, standard deviation, difference pre-post in \%, effect sizes pre-post and standard error).

Data Sheet 4 | Jump high pre-post for CG and EG (mean, standard deviation, difference pre-post in \%, effect sizes pre-post and standard error).

\section{REFERENCES}

Amaro-Gahete, F. J., De-La, O. A., Sanchez-Delgado, G., Robles-Gonzalez, L., Jurado-Fasoli, L., Ruiz, J. R., et al. (2018). Whole-body electromyostimulation improves performance-related parameters in runners. Front. Physiol. 9:1576. doi: 10.3389/fphys.2018.01576

Babault, N., Cometti, G., Bernardin, M., Pousson, M., and Chatard, J. C. (2007). Effects of electromyostimulation training on muscle strength and power of elite rugby players. J. Strength Cond. Res. 21, 431-437. doi: 10.1519/00124278-200705000-00025

Bax, L., Staes, F., and Verhagen, A. (2005). Does neuromuscular electrical stimulation strengthen the quadriceps femoris? A systematic review of randomised controlled trials. Sports Med. 35, 191-212. doi: 10.2165/00007256-200535030-00002

Billot, M., Martin, A., Paizis, C., Cometti, C., and Babault, N. (2010). Effects of an electrostimulation training program on strength, jumping, and kicking capacities in soccer players. J. Strength Cond. Res. 24, 1407-1413. doi: 10.1519/JSC.0b013e3181d43790

Borenstein, M., Hedges, L. V., Higgins, J. P., and Rothstein, H. R. (2010). A basic introduction to fixed-effect and random-effects models for meta-analysis. Res. Synth. Methods 1, 97-111. doi: 10.1002/jrsm.12

Brocherie, F., Babault, N., Cometti, G., Maffiuletti, N., and Chatard, J. C. (2005). Electrostimulation training effects on the physical performance of ice hockey players. Med. Sci. Sports Exerc. 37, 455-460. doi: 10.1249/01.MSS.0000155396.51293.9F

Cohen, J. (1988). Statistical Power Analysis for the Behavioral Sciences. Hillesdale NJ: Lawrence Erlbaum Associates.

Deeks, J. J., and Higgins, J. P. (2010). Statistical Algorithms in Review Manager 5. Statistical Methods Group of the Cochrane Collaboration, 1-11. Available online at: https://training.cochrane.org/handbook/statisticalmethods-revman5 (accessed October 14, 2019).

Delitto, A., Brown, M., Strube, M. J., Rose, S. J., and Lehman, R. C. (1989). Electrical stimulation of quadriceps femoris in an elite weight lifter: a single subject experiment. Int. J. Sports Med. 10, 187-191. doi: 10.1055/s-2007-1024898

Dörmann, U. (2011). Isometrische und Isoinertiale Parameter in der Kraftdiagnostik: Reliabilitätsprüfung und Evaluation von Effekten Mechanischer und Elektrischer Krafttrainingsreize. (Dissertation). Cologne, Germany.

Dörmann, U., Houben, P., Ziadi, S., Nowak, S., Wirtz, N., Kleinoder, H., et al. (2011). "Effects of dynamic electromyostimulation of the leg muscle chain on isometric and isoinertial strength parameters and sprint performance," in 16th Annual Congress of the European College of Sport Science, eds T. Cable and K. George (Liverpool), 524. 
Dörmann, U., Wirtz, N., Micke, F., Morat, M., Kleinöder, H., and Donath, L. (2019). The Effects of superimposed whole-body electromyostimulation during short-term strength training on physical fitness in physically active females: a randomized controlled trial. Front. Physiol. 10:728. doi: $10.3389 /$ fphys.2019.00728

Filipovic, A., De Marées, M., Grau, M., Hollinger, A., Seeger, B., Schiffer, T., et al. (2019). Superimposed whole-body electrostimulation augments strength adaptations and Type II myofiber growth in soccer players during a competitive season. Front. Physiol. 10:1187. doi: 10.3389/fphys.2019.01187

Filipovic, A., Grau, M., Kleinoder, H., Zimmer, P., Hollmann, W., and Bloch, W. (2016). Effects of a whole-body electrostimulation program on strength, sprinting, jumping, and kicking capacity in elite soccer players. J. Sports Sci. Med. 15, 639-648.

Filipovic, A., Kleinoder, H., Dormann, U., and Mester, J. (2012). Electromyostimulation-a systematic review of the effects of different electromyostimulation methods on selected strength parameters in trained and elite athletes. J. Strength Cond. Res. 26, 2600-2614. doi: 10.1519/JSC.0b013e31823f2cd1

Glatthorn, J. F., Gouge, S., Nussbaumer, S., Stauffacher, S., Impellizzeri, F. M., and Maffiuletti, N. A. (2011). Validity and reliability of optojump photoelectric cells for estimating vertical jump height. J. Strength Cond. Res. 25, 556-560. doi: 10.1519/JSC.0b013e3181ccb18d

Goode, A. P., Reiman, M. P., Harris, L., Delisa, L., Kauffman, A., Beltramo, D., et al. (2015). Eccentric training for prevention of hamstring injuries may depend on intervention compliance: a systematic review and meta-analysis. Br. J. Sports Med. 49, 349-356. doi: 10.1136/bjsports-2014-093466

Green, B. S., Blake, C., and Caulfield, B. M. (2011). A valid field test protocol of linear speed and agility in rugby union. J. Strength Cond. Res. 25, 1256-1262. doi: 10.1519/JSC.0b013e3181d8598b

Gregory, C. M., and Bickel, C. S. (2005). Recruitment patterns in human skeletal muscle during electrical stimulation. Phys. Ther. 85, 358-364. doi: 10.1093/ptj/85.4.358

Herrero, A. J., Martín, J., Martín, T., Abadía, O., Fernández, B., and García-López, D. (2010a). Short-term effect of plyometrics and strength training with and without superimposed electrical stimulation on muscle strength and anaerobic performance: a randomized controlled trial. Part II. J. Strength Cond. Res. 24, 1616-1622. doi: 10.1519/JSC.0b013e3181d8e84b

Herrero, A. J., Martin, J., Martin, T., Abadia, O., Fernandez, B., and GarciaLopez, D. (2010b). Short-term effect of strength training with and without superimposed electrical stimulation on muscle strength and anaerobic performance. A randomized controlled trial. Part I. J. Strength Cond. Res. 24, 1609-1615. doi: 10.1519/JSC.0b013e3181dc427e

Herrero, J. A., Izquierdo, M., Maffiuletti, N. A., and Garcia-Lopez, J. (2006). Electromyostimulation and plyometric training effects on jumping and sprint time. Int. J. Sports Med. 27, 533-539. doi: 10.1055/s-2005865845

Kastner, A., Braun, M., and Meyer, T. (2015). Two cases of rhabdomyolysis after training with electromyostimulation by 2 young male professional soccer players. Clin. J. Sport Med. 25, e71-73. doi: 10.1097/JSM.0000000000000153

Kemmler, W., Fröhlich, M., Stengel, S., and Kleinöder, H. (2016). Wholebody electromyostimulation - the need for common sense! Rationale and guideline for a safe and effective training. Dtsch. Z. Sportmed. 67, 218-221. doi: 10.5960/dzsm.2016.246

Kemmler, W., Von Stengel, S., Schwarz, J., and Mayhew, J. L. (2012). Effect of whole-body electromyostimulation on energy expenditure during exercise. J. Strength Cond. Res. 26, 240-245. doi: 10.1519/JSC.0b013e31821a3a11

Kemmler, W., Weissenfels, A., Willert, S., Shojaa, M., Von Stengel, S., Filipovic, A., et al. (2018). Efficacy and safety of low frequency wholebody electromyostimulation (WB-EMS) to improve health-related outcomes in non-athletic adults. A systematic review. Front. Physiol. 9:573. doi: $10.3389 /$ fphys.2018.00573

Kots, Y., and Chwilon, W. (1971). Muscle Training with the Electrical Stimulation Method. Teoriya i Praktika Fizicheskoi Kultury.

Maffiuletti, N. A., Bramanti, J., Jubeau, M., Bizzini, M., Deley, G., and Cometti, G. (2009). Feasibility and efficacy of progressive electrostimulation strength training for competitive tennis players. J. Strength Cond. Res. 23, 677-682. doi: 10.1519/JSC.0b013e318196b784
Maffiuletti, N. A., Cometti, G., Amiridis, I. G., Martin, A., Pousson, M., and Chatard, J. C. (2000). The effects of electromyostimulation training and basketball practice on muscle strength and jumping ability. Int. J. Sports Med. 21, 437-443. doi: 10.1055/s-2000-3837

Maffiuletti, N. A., Herrero, A. J., Jubeau, M., Impellizzeri, F. M., and Bizzini, M. (2008). Differences in electrical stimulation thresholds between men and women. Ann. Neurol. 63, 507-512. doi: 10.1002/ana.21346

Malatesta, D., Cattaneo, F., Dugnani, S., and Maffiuletti, N. A. (2003). Effects of electromyostimulation training and volleyball practice on jumping ability. J. Strength Cond. Res. 17, 573-579. doi: 10.1519/00124278-200308000-00025

Micke, F., Kleinöder, H., Dörmann, U., Wirtz, N., and Donath, L. (2018). Effects of an eight-week superimposed submaximal dynamic whole-body electromyostimulation training on strength and power parameters of the leg muscles: a randomized controlled intervention study. Front. Physiol. 9:1719. doi: 10.3389/fphys.2018.01719

Nuzzo, J. L., and McBride, J. M. (2013). The effect of loading and unloading on muscle activity during the jump squat. J. Strength Cond. Res. 27, 1758-1764. doi: 10.1519/JSC.0b013e318291b8b2

Paillard, T. (2008). Combined application of neuromuscular electrical stimulation and voluntary muscular contractions. Sports Med. 38, 161-177. doi: 10.2165/00007256-200838020-00005

Requena Sánchez, B., Padial Puche, P., and Gonzalez-Badillo, J. J. (2005). Percutaneous electrical stimulation in strength training: an update. J. Strength Cond. Res. 19, 438-448. doi: 10.1519/00124278-200505000-00033

Stojanovic, E., Ristic, V., McMaster, D. T., and Milanovic, Z. (2017). Effect of plyometric training on vertical jump performance in female athletes: a systematic review and meta-analysis. Sports Med. 47, 975-986. doi: 10.1007/s40279-016-0634-6

Tiggemann, C. L., Korzenowski, A. L., Brentano, M. A., Tartaruga, M. P., Alberton, C. L., and Kruel, L. F. M. (2010). Perceived exertion in different strength exercise loads in sedentary, active, and trained adults. J. Strength Cond. Res. 24, 2032-2041. doi: 10.1519/JSC.0b013e3181d32e29

Vanderthommen, M., and Duchateau, J. (2007). Electrical stimulation as a modality to improve performance of the neuromuscular system. Exerc. Sport Sci. Rev. 35, 180-185. doi: 10.1097/jes.0b013e318156e785

Voelzke, M., Stutzig, N., Thorhauer, H. A., and Granacher, U. (2012). Promoting lower extremity strength in elite volleyball players: effects of two combined training methods. J. Sci. Med. Sport 15, 457-462. doi: 10.1016/j.jsams.2012.02.004

Westing, S. H., Seger, J. Y., and Thorstensson, A. (1990). Effects of electrical stimulation on eccentric and concentric torque-velocity relationships during knee extension in man. Acta Physiol. Scand. 140, 17-22. doi: 10.1111/j.1748-1716.1990.tb08971.x

Wirtz, N., Zinner, C., Doermann, U., Kleinoeder, H., and Mester, J. (2016). Effects of loaded squat exercise with and without application of superimposed EMS on physical performance. J. Sports Sci. Med. 15, 26-33.

Wolf, S. L., Ariel, G. B., Saar, D., Penny, M. A., and Railey, P. (1986). The effect of muscle stimulation during resistive training on performance parameters. Am. J. Sports Med. 14, 18-23. doi: 10.1177/036354658601400104

Young, W. B. (2006). Transfer of strength and power training to sports performance. Int. J. Sports Physiol. Perform. 1, 74-83. doi: 10.1123/ijspp.1.2.74

Zink, A. J., Whiting, W. C., Vincent, W. J., and McLaine, A. J. (2001) The effects of a weight belt on trunk and leg muscle activity and joint kinematics during the squat exercise. J. Strength Cond. Res. 15, 235-240. doi: 10.1519/00124278-200105000-00013

Conflict of Interest: The authors declare that the research was conducted in the absence of any commercial or financial relationships that could be construed as a potential conflict of interest.

Copyright (c) 2019 Wirtz, Dörmann, Micke, Filipovic, Kleinöder and Donath. This is an open-access article distributed under the terms of the Creative Commons Attribution License (CC BY). The use, distribution or reproduction in other forums is permitted, provided the original author(s) and the copyright owner(s) are credited and that the original publication in this journal is cited, in accordance with accepted academic practice. No use, distribution or reproduction is permitted which does not comply with these terms. 